\title{
Devising a Game Theoretic Approach to Enable Smart City Digital Twin Analytics
}

\author{
Neda Mohammadi \\ Georgia Institute of Technology \\ nedam@gatech.edu
}

\section{Abstract}

Despite investments in advancing information and communications technology (ICT)-integrated infrastructure systems toward becoming Smarter Cities, cities often face a large gap between smart sustainable supply and demand. Here, we review the core concepts of ICT-integrated infrastructure systems as they pertain to developing smart and sustainable cities, and describe how a game theoretic-based digital twin of a city can enable more visibility and insight into the successful implementation of such systems. This study is a foundational step toward enabling participation of all city stakeholders (i.e., government, industry, and citizens) in the decision making process and the creation of smart sustainable cities. Engaging city stakeholders in such a manner allows for collective participation in changes, which can enable continuous adaptation toward more sustaining growth and prosperity.

\section{Smart Sustainable Cities}

\subsection{Urbanization, Growth of Supply and Demand, and Urge for Efficiency}

Between 1950 and 2018, the world's urban population have grown from 751 to more than 4.2 billion. Projections anticipate that, by 2050 , they will constitute nearly $70 \%$ of the world population [1]. In the United States, currently the most urbanized region in the world, this percentage is expected to increase to $90 \%$ [1].

As the world continues to urbanize, the increasing day-to-day service demand of citizens, embedded in a growing socio-economic structure, requires a new paradigm of supply infrastructure. Unlike biological systems where growth, due to terminal biological imbalance between supply and demand of the metabolic system, is ultimately limited to an asymptotic body size [2]; growth of cities, due to continuous socio-economic interactions that generate a feedback loop, is considered

\author{
John E. Taylor \\ Georgia Institute of Technology \\ jet@gatech.edu
}

to be unbounded [3]. While the socio-economic quantities such as wage, gross domestic product (GPD), innovation, crime, traffic congestion, disease, etc. increase superlinearly by about $15 \%$, the demand in growth of city infrastructure systems opportunely scales sublinearly with an exponent of approximately 0.85 $[3,4]$. Considering the immense strain that is placed on resources due to rising urban population, and that much of physical infrastructure is built with a lifespan of 30-50 years, increasing the efficiency of infrastructure services has become an imperative to sustaining growth of cities. Research has suggested that cities must increase resource efficiency by factors of 4 to 10 , through multi-sector infrastructure interventions, which can yield the highest efficiency gains, in order to cope with the increasing demand and complexities [5]. Thereby, cities have begun integrating information and communications technology (ICT) with their traditional infrastructure, establishing themselves as smarter cities [6]-for further theoretical definition on smart cities refer to $[6,7,8,9]$. Leveraging ICT to establish smarter supply infrastructure is no longer a luxury, but a necessity, if we are to accommodate accelerating growth and create smart sustainable cities [10], which may otherwise lead to resource scarcity, socio-economic degradation, or, at the extreme, complete collapse. Sustaining the growth of cities in all dimensions, thus, requires resource efficiency and continuous adaptation of infrastructure systems in response to socio-economic feedback loops, which may be attainable through technological paradigm shifts $[3,11]$.

\subsection{ICT Solutions and Implementation Gap}

Harnessing ICT to advance the efficiency of city infrastructure service delivery was promulgated by IBM with the objective of improving management of services in partnership with city governments [12, 13]. A number of technology companies, such as Cisco, IBM, and Siemens, were the early adopters of the term 'smart cities' [14]. Integrating their 
smart consultation services with their data analytics capacities to optimize urban systems, IBM established the Smarter Planet [15] initiative, focused on ICT-driven urban change towards Smarter Cities [16]. The IBM Smarter City Challenge [17], aims to address unique problems specified by cities across nine pillars of Administration, Citizen Engagement, Economic Development, Education \& Workforce, Environment, Public Safety, Social Services, Transportation, and Urban Planning. It has since enabled many service management data analytics platforms for municipalities such as: an energy efficiency and solar technology-based power distribution system for advancing energy efficiency, and a Smart City Control System for Masdar City to address the challenge of prioritizing users in energy distribution [18]; Rio de Janeiro's Urban Operations Center in preparation for the 2016 Summer Olympic Games, which includes live weather and street-level video feeds combined with social media, crime and security monitoring and analytics [19]; and Boulder, Colorado's energy utility system including smart energy meters and implementation of a smart electricity grid, which was mainly a technology and analytics driven solution without significant input from the public [20]. Nevertheless, since the beginning of the program in 2009, cities have joined the challenge with incentives beyond efficiency and citizen satisfaction. Surprisingly, many cities were joining the challenge to market their capacity for economic growth and competitiveness at the global level and to position themselves as 'innovative cities' to attract the 'Creative Class' of the new generation of workforce, especially in the middle of the 2008-09 economic crisis [21, 14]. This has resulted in poor implementation of ICT-based development strategies by many city governments that are merely limited to "attractive and tangible facilities such as public wireless networks, electric vehicle charging stations, bicycle lanes, and so forth" [14].

Socio-economic challenges that impeded technology implementation such as, safety and security, decentralization of government, migration, and social equity etc., quickly hindered the data-driven approaches and overrode IBM's vision in some of the projects (e.g., the Digital On-Ramps), which were later recognized as 'global urban trends' that are affecting many cities around the world today $[17,22]$. Therefore, recognizing disconnects between existing smart cities concepts and initiatives versus successful implementation efforts is of utmost importance [16]. Just as lack of technological development and innovation can lead to collapse, inappropriate implementation of new systems can lead to failure [23, 24]. This results from implementing ICT-based applications in the name of smart digitization without engaging impacted sectors and entities. Independent automation of infrastructure in hopes of improved efficiency, or independent enhancement of government management capacity in hopes of improved economy, may not sustain without stakeholder engagement. In reality, a utopian smart city which is instrumented, interconnected, and intelligent such that all systems communicate and are optimized [15], needs to co-exist and co-evolve with the underlying dynamic socio-economic structure.

As specified by the body of research that studies cities as complex adaptive systems [25, 26, 27, 28] that are extremely diverse, dynamic, and prone to change, very simple underlying dynamics may result in large spatiotemporal fluctuations and potentially lead to chaos. Hence, continuous adaptation of strategies integrated with clear pathways to implementation and engagement of the citizens, as a critical part of the socio-economic structure, are key elements in the path toward sustaining growth. If the socio-economic interactions are effectively achieved in space and time, cities can become accelerated concentrations of social activities [25].

In the existing efforts to integrate ICT with traditional physical infrastructure to enable smarter cities, however, we lack appropriate integration of socio-economic, and more broadly, the human dynamics of cities. Here, we explore how integrating cities' Human-Infrastructure-Technology interactions and harnessing socio-economic feedback loops may serve as a dynamic decision making platform for continuous adaptation. In particular, we explore whether a game theoretic approach will prove valuable in effectively integrating the key operative elements of a smart city in its digital twin analytics platform. We explore this through the illustrative case of an autonomous vehicle transit smart city plan in a major U.S. city.

\section{The Game of Smart City Digital Twins}

\subsection{Human, Infrastructure, and Technology Interdependence}

Engineering systems are in essence placing a combination of components together such that they can synergistically function and collectively achieve a desired objective. In the case of cities as systems of systems, however, unlike most deterministic engineered systems the collective behavior is known to be different than the sum of its parts. Such behavior stems from the interdependencies between human-infrastructure-technology elements and largely 


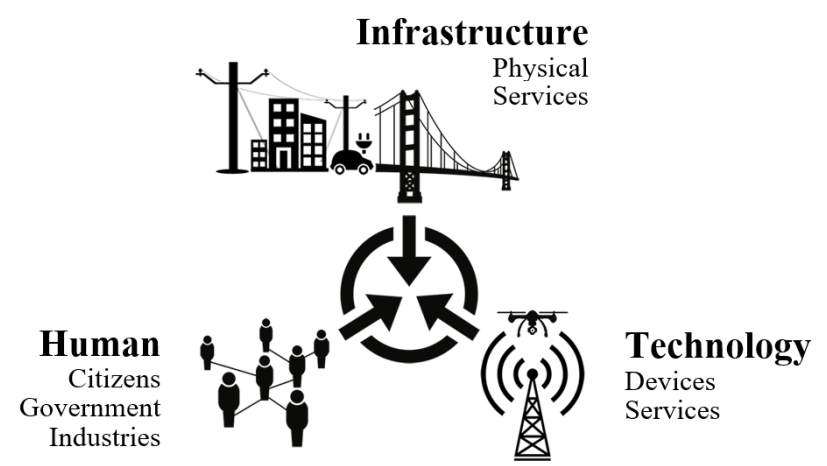

Figure 1. Integration of Humans, Infrastructure, and Technology in decision making.

relies on how these components interact, condition, and constrain each other over time and space. Sound mathematical models generated through sensor-based data may not be able to reveal the full spectrum of such causal relationships, or circular causalities [25], such as when individuals change their time and location designations in a heterogeneous manner. Scientific predictions over large spatiotemporal scales is difficult. Even prior to the technological advancements of today, the intricate interdependencies between infrastructure and socio-economic elements have made cities nearly impossible to fully simulate [29]. Therefore, in order for reliable interpretation, the interdependencies need to be locally and temporally contextualized.

At the highest level of abstraction, a city can be classified into the components identified in Figure 1: (1) Human: comprising the city stakeholders, namely government (i.e., governing organizations and policy makers), industry (i.e., vendors and manufacturing, infrastructure service providers, food and healthcare, trade, financial and insurance, information, media and technology, etc.), and citizens (can be viewed as users of technology or consumers of the infrastructure services); (2) Infrastructure: including both the physical infrastructure and the infrastructure services that they provide broadly divided into buildings, mobility, energy, water/waste, and communication; and (3) Technology: incorporating devices and services (i.e., sensors and other data generation and communication devices, data storage and transmission, management, and analytics infrastructure).

\subsection{Space-time Dynamics and Digital Twins}

The conception of Digital Twins [30, 31, 32, 33] in engineering may date back to the emergence of object oriented computer-aided design (CAD) systems that revolutionized the design process for engineers and architects from old forms of physical prototyping into digital prototyping. Although CAD enabled interactive and direct modeling by providing the ability to digitally model the associative geometric relationships, it was still limited to a static model. Relatively recently, with the ability to fuse data into the digital prototype, we are able to model behavior beyond geometry and create dynamic models that can integrate real world conditions [34, 35]. Such thinking has inspired the idea of creating intelligent adaptive machines in manufacturing by developing a digital duplicate (aka digital twin) of the real system that parallels its behavior in real-time. In the simplest terms, a digital twin has the potential to enable real-time monitoring and seamless analytics and simulations across time and space. As depicted in Figure 2, once applicable interdependencies are modelled and fused with reality data, the behavior of the systems can be monitored at any state $t_{0}\left(x_{0}, y_{0}\right)$. From this space-time origin, alternative states (real, or hypothetical) may be examined by navigating in time and space $t_{i}\left(x_{i}, y_{i}\right)$. The system can recall an event from the past, simulate hypothetical 'what if?' scenarios in the past, predict states with the highest probability in the future, or simulate in what conditions a hypothetical 'what if?' scenario is probable in the future.

NASA [36] was among the early adopters of the digital twin concept in its Apollo program. Among the major players of the digital twin market are currently GE's Predix Platform [37], IBM's Watson new Platform capabilities around Digital Twin [37], Siemens Digital Innovation Platform [38], Microsoft's vision for digital twins as a 'process digital twin' and achieving operational excellence through digital twin strategy and platforms [39], alongside the efforts of consulting companies such as McKinsey \& Company [38] and Deloitte's [40] Industry 4.0 visions for digital manufacturing. Notwithstanding this adoption by industry, we lacks rigorous supporting scientific research in this area, which raises concerns for successful implementation at the city level. 
Creating Smart City Digital Twins [41](Figure 3), for non-deterministic complex adaptive systems of cities, will be most effective if they can bring visibility and insight into how the nature of change can be influenced and led towards sustaining growth [42]. Although capturing the dynamic interdependencies of human-infrastructure-technology and harnessing the socio-economic feedback loops are not trivial, the promise of today's connectivity and analytics capabilities enabled by various technologies, such as internet of things (IoT), enables a new theoretical framework for the study of smart cities.

In a city viewed as a complex system, where space-time dependencies of events and decisions are non-deterministic and dynamic, a digital twin may be able to virtually capture interdependencies between infrastructure performance, technology interventions, and human dynamics. When cognizant of the city's spatiotemporal dynamics, it can progressively become more capable of predicting future probabilities, as well as simulating hypothetical 'what if?' scenarios. Understanding the nature of change as these entities interact with one another over time will guide future strategies by assessing whether or not smart growth strategies are effective. It can identify the drivers of possible disruption and minimize the gap between smart utopia and smart reality [43]. The ability to process and examine strategic states when decision-makers interact, can be embedded in the smart city digital twin, which can then be used to support and enable game theoretic analyses and simulation capabilities.

\subsection{A Game-theoretic Approach to Decision Making}

Game theoretic decision making related to Smart City Digital Twins, at the highest level, entails participation of three stakeholders (i.e., government, industry, and citizens). The payoff for smart sustainable city interventions for each group of stakeholders are distinct from one another. While the government is concerned with and values aspects such as cost, citizen satisfaction, and the city's attractiveness and competitiveness, industry is interested in payoffs in the form of profitability, return on investment (ROI), and fostering a relationship with customers. Nonetheless, citizens tend to largely attend to values such as savings and expenses, quality, access, equity, employment, education, culture, and health.

The game is built around the regulations involved with the overall makeup of the city defined by the interdependencies between human-infrastructure-technology. Sustaining growth of the city is the desired emerging outcome of the game. At any n-actor intervention $\langle N, S, F\rangle$ :

$N=\{1, \ldots, n\}$

$\boldsymbol{S}=S_{1}, S_{2}, \ldots, S_{n} \in S=S_{1} \times \ldots \times S_{n}$

$\boldsymbol{F}(H, I, T)=F_{1}, F_{2}, \ldots, F_{n} \in F=F_{1} \times \ldots \times F_{n}$

for actor $i$ :

$F_{i}: S=S_{1} \times \ldots \times S_{n} \rightarrow \mathbb{R}$

where $N$ is a set of $n$ actors (indexed by $i$ ), $S$ is a strategy set, $F$ is a utility function set, and $H, I$, and $T$ are the human, infrastructure, and technology variables.

A prominent example is the Let's Move Nashville autonomous vehicles transit plan for Nashville, one of the fastest growing metropolitan areas in the U.S. with an expected 1.0M increase in population by 2040 , in which the regional mobility is a critical challenge. The nMotion plan is a strategic regional transit plan with the objective of enhancement, simplification, and sustainment of transportation network over time and identifies opportunities for growing mobility. The strategy involves exploring the needs and preferences of the citizens and general receptivity of three alternative transit solutions (i.e., comprehensive regional system,

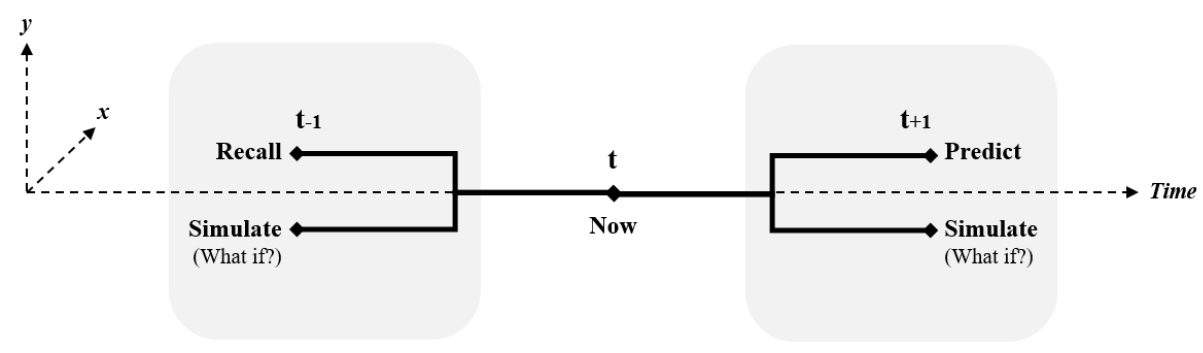

Figure 2. Digital Twin: monitoring, analytics, and simulation across time and space. 
bus system expansion, and modest improvements) through online surveys. Interestingly, in spite of the of the viable roadway improvement and capacity expansion solutions, the bold long term alternative strategy for paradigm shifting transit technology such as lightweight transit, rapid bus and autonomous vehicles integrated with pedestrian and cycling capacities was chosen by the citizens [44]. Implementation of this project is non-trivial and involves ongoing collaboration of many stakeholders and communities. Therefore, a trade-off exists between investing in additional infrastructure or additional ICT, the pay-offs of which vary for the political, industry, and citizen stakeholders.

\subsubsection{Competition and Nash Equilibrium.} Competitive (non-cooperative) strategic situations in a Smart City Digital Twin game are largely concerned with advancing the micro-level 'smartness' of the city. This involves ICT-based developments with the objective of improving efficiency, and elevating the cities' innovation and competitiveness. Such competitive interactions among the three stakeholders are monitored across time to examine whether or not the selected courses of action are maximizing benefits to all actors according to the Nash equilibrium. Nash equilibrium ensures that no actor $i$ can unilaterally improve their utility function, while the strategies of other actors are fixed, Eq.(1) [45]. The smart cities, in which we intend to incorporate historical information in time and space into our decision making, however, is a dynamic game. This state characterizes a vector of probabilities of the strategy sets, and can result in multiple Nash equilibria. Selecting the optimal equilibrium, is therefore, highly dependent on the quality of our historical information embedded in the utility function. Such analysis could be useful in understanding and predicting the outcome of the Let's
Move Nashville autonomous vehicle transit plan in Nashville.

$$
F_{i}\left(S_{i}^{*}, S_{-i}^{*}\right) \geq\left(S_{i}, S_{-i}^{*}\right), \forall S_{i} \in S
$$

\subsubsection{Cooperation and the Shapley Value.}

non-cooperative games, in which the actors are assumed not to have direct coordination or communication with one another, the cooperative strategic situation in a smart city digital twin game largely involves continuous coalition of all three stakeholders towards longer term objectives such as sustainability, resilience, and sustaining growth. In such conditions, cost-benefit equations should be decomposed across time and space such that situations of interdependence between stakeholders lead to desired macro-level dynamics that are aligned with sustainability and resilience objectives determined by the focal points that are incentive compatible (e.g., the Shapley Value, in which the average marginal contribution of actor $i$ is captured by assigning a probability weight towards building the grand coalition $S$ ). Here, the actors often benefit from shared utility functions, and decide to fully cooperate. smart city digital twins can evaluate 'what if?' scenarios by examining incentives to promote cooperation [46]. This implies sustainment of the Let's Move Nashville and its implications of the sustainability and growth of the city in the longer term.

\subsection{Virtualization, Envisioning 'What if?' Scenarios, and Citizen Engagement}

Although mathematical models can perform probabilistic simulations and explain trends by reciprocal spatiotemporal data exchange between the city and its digital twin (Figure 3), capturing the true state of condition requires context. Virtual Reality (VR), Augmented Reality (AR), and Mixed Reality (MR)

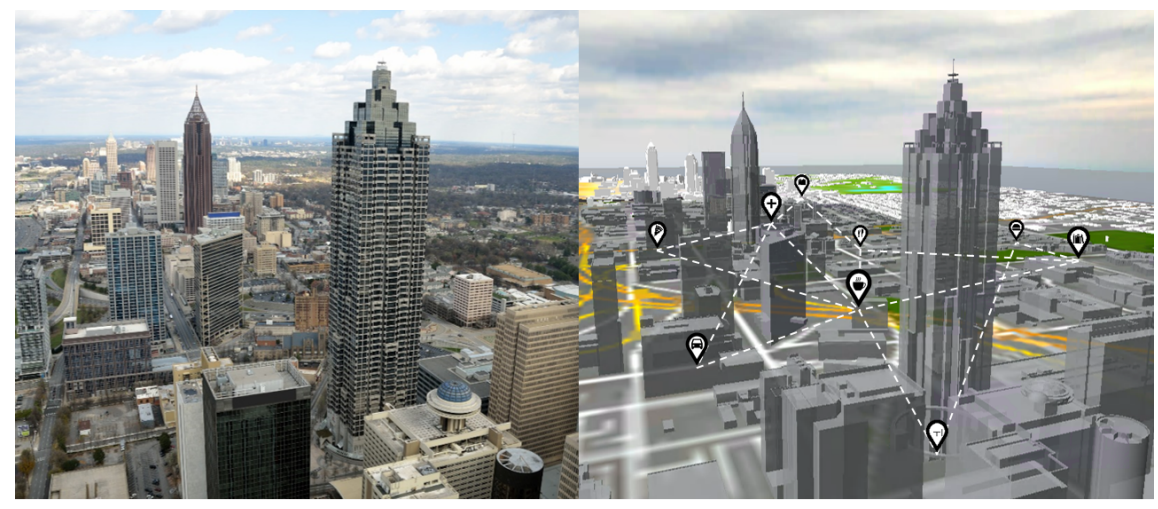

Figure 3. Smart City Digital Twin: City of Atlanta. 
offer an ideal opportunity for capturing such nuances, and have the ability to extend the space-time domain to an additional context dimension. This is particularly important when engaging the non-expert citizen into the decision making process. Citizen's access to the digital twin platform through online and mobile applications can become a conduit for crowd-sensing [44] and increased visibility and input from the public. This could facilitate continuous participation of citizens and communities, especially in long term projects such as Let's Move Nashville.

\section{Leading Change toward Sustaining Growth}

The conception of transforming cities to Smart Cities [8] may have originally gained momentum after the Smart Growth [47] movement in the late 1990s, which initially reinforced the need for new policies for urban planning. Today, whether named digital cities [48, 49], intelligent cities [48], virtual cities [50], utopian cities [51], innovative cities [52], competitive cities [53], responsive cities [54], resilient cities [55], sustainable cities [56], or mere corporate storytelling [57], cities around the world are advocating for better quality of life [58] for their citizens. Closing the gap between the growth in population and growth in efficiency, productivity, sustainability, resilience, competitiveness, and quality of life is only possible with the engagement of all city stakeholders. If strategic considerations are collectively determined and led by the stakeholders, we may start observing effective adaptation leading to smart growth as seen in some cities such as Portland and Los Angeles [52].

The United Nations (UN) places successful management of urban growth-closely related to the three dimensions of sustainable development, namely: economic, social, and environmental-at the center of the sustainable development goals (SDGs) agenda [1]. Pro-poor, equitable, and scalable technology and innovation is needed [1]. Social sustainability challenges such as neighborhood inequalities [59], are compromising the capacity of cities to collectively gain growth. Today, thanks to the advancement of technologies such as MEMS, Wi-Fi, AMI, RFID, etc., we have the ability to measure and sense the space time conditions of myriad aspects of a city. The real challenge lies in making coalition cases integrated across human-infrastructure-technology and succeeding in implementation. The National Academies of Sciences, Engineering, and Medicine recommend that cities establish their local sustainability plans and decision making as every region has its own unique challenges that requires broad stakeholder engagement in developing and implementing actions [60].

The rate of theoretical and scientific discovery is not keeping pace with the rate of technology development and industry investments. Smart city digital twins may enable an ongoing conversation which, in turn, facilitates smart interventions. Such interdisciplinary collaborations may bring these perspectives into focus, largely fashioned towards sustaining growth and advancing the quality of life in cities.

\section{Acknowledgement}

This study was supported in part by the Graphics, Visualization, and Usability (GVU) Center and the Institute for People and Technology (IPaT) at the Georgia Institute of Technology.

\section{References}

\section{References}

[1] United Nations, "World Urbanization Prospects: The 2018 Revision," 2018.

[2] G. B. West, J. H. Brown, and B. J. Enquist, "A general model for ontogenetic growth," Nature, vol. 413, no. 6856, p. $628,2001$.

[3] L. Bettencourt and G. West, "A unified theory of urban living," Nature, vol. 467, no. 7318, p. 912, 2010.

[4] G. West, Scale: The Universal Laws of Growth, Innovation, Sustainability, and the Pace of Life in Organisms, Cities, Economies, and Companies. Penguin, 2017.

[5] A. Ramaswami, A. G. Russell, P. J. Culligan, K. R. Sharma, and E. Kumar, "Meta-principles for developing smart, sustainable, and healthy cities," Science, vol. 352, no. 6288, pp. 940-943, 2016.

[6] M. Batty, K. W. Axhausen, F. Giannotti, A. Pozdnoukhov, A. Bazzani, M. Wachowicz, G. Ouzounis, and Y. Portugali, "Smart cities of the future," The European Physical Journal Special Topics, vol. 214, no. 1, pp. 481-518, 2012.

[7] V. Albino, U. Berardi, and R. M. Dangelico, "Smart cities: Definitions, dimensions, performance, and initiatives," Journal of Urban Technology, vol. 22, no. 1, pp. 3-21, 2015.

[8] H. Chourabi, T. Nam, S. Walker, J. R. Gil-Garcia, S. Mellouli, K. Nahon, T. A. Pardo, and H. J. Scholl, "Understanding smart cities: An integrative framework," in System Science (HICSS), 2012 45th Hawaii International Conference on, pp. 2289-2297, IEEE, 2012.

[9] A. Glasmeier and S. Christopherson, "Thinking about smart cities," 2015.

[10] S. E. Bibri and J. Krogstie, "Smart sustainable cities of the future: An extensive interdisciplinary literature review," Sustainable Cities and Society, vol. 31, pp. 183-212, 2017.

[11] S. Milgram, "The experience of living in cities," Science, vol. 167, no. 3924, pp. 1461-1468, 1970. 
[12] C. Harrison, B. Eckman, R. Hamilton, P. Hartswick, J. Kalagnanam, J. Paraszczak, and P. Williams, "Foundations for Smarter Cities," 2010.

[13] Samuel J. Palmisano, "Welcome to the Decade of Smart," tech. rep., IBM, London, 2010.

[14] C. Harrison and I. A. Donnelly, "A theory of smart cities," in Proceedings of the 55th Annual Meeting of the ISSS-2011, Hull, UK, vol. 55, 2011.

[15] Samuel J. Palmisano, "A Smarter Planet: The Next Leadership Agenda," 2008

[16] Susanne Dirks and Mary Keeling, "A vision of smarter cities," tech. rep., IBM, New York, NY, USA, 2009.

[17] IBM, "IBM Smarter Cities Challenge," 2018.

[18] F. Cugurullo, "How to build a sandcastle: An analysis of the genesis and development of Masdar City," Journal of Urban Technology, vol. 20, no. 1, pp. 23-37, 2013.

[19] R. Goodspeed, "Smart cities: moving beyond urban cybernetics to tackle wicked problems," Cambridge Journal of Regions, Economy and Society, vol. 8, no. 1, pp. 79-92, 2014.

[20] IBM, "IBM's Smarter Cities Challange: Boulder Report," tech. rep., IBM, 2011.

[21] A. Wiig, "IBM's smart city as techno-utopian policy mobility," City, vol. 19, no. 2-3, pp. 258-273, 2015.

[22] UN Human Settlements Program (UN Habitat), "World Cities Report," tech. rep., 2016.

[23] J. Markard, "Transformation of infrastructures: sector characteristics and implications for fundamental change," Journal of Infrastructure Systems, vol. 17, no. 3, pp. 107-117, 2011.

[24] C. Christensen, The innovator's dilemma: when new technologies cause great firms to fail. Harvard Business Review Press, 2013.

[25] L. M. A. Bettencourt, "Cities as complex systems," Modeling complex systems for public policies, pp. 217-238, 2015.

[26] M. Batty, Cities and complexity: understanding cities with cellular automata, agent-based models, and fractals. The MIT press, 2007.

[27] K. R. Malik, Y. Sam, M. Hussain, and A. Abuarqoub, "A methodology for real-time data sustainability in smart city: Towards inferencing and analytics for big-data," Sustainable Cities and Society, vol. 39, pp. 548-556, 2018.

[28] N. Kumar, N. Chilamkurti, and S. C. Misra, "Bayesian coalition game for the internet of things: an ambient intelligence-based evaluation," IEEE Communications Magazine, vol. 53, no. 1, pp. 48-55, 2015.

[29] M. Fuller and R. Moore, The death and life of great American cities. Macat Library, 2017.

[30] M. Grieves, "Digital twin: Manufacturing excellence through virtual factory replication," tech. rep., 2014.

[31] GE, "Mind + Machine: What is a Digital Twin?," 2017.

[32] S. P. A. Datta, "Emergence of Digital Twins," arXiv preprint arXiv:1610.06467, 2016.

[33] S. Boschert and R. Rosen, "Digital twin-the simulation aspect," in Mechatronic Futures: Challenges and Solutions for Mechatronic Systems and Their Designers, pp. 59-74, 2016.
[34] A. Francisco, H. Truong, A. Khosrowpour, J. E Taylor, and N. Mohammadi, "Occupant perceptions of building information model-based energy visualizations in eco-feedback systems," Applied Energy, vol. 221, pp. 220-228, 2018.

[35] M. Grieves and J. Vickers, "Digital twin: Mitigating unpredictable, undesirable emergent behavior in complex systems," in Transdisciplinary Perspectives on Complex Systems, pp. 85-113, Springer, 2017.

[36] E. Glaessgen and D. Stargel, "The digital twin paradigm for future NASA and US Air Force vehicles," in 53rd AIAA/ASME/ASCE/AHS/ASC Structures, Structural Dynamics and Materials Conference 20th AIAA/ASME/AHS Adaptive Structures Conference 14th AIAA, p. 1818, 2012.

[37] GE, "Predix," 2018.

[38] McKinsey \& Company, "Developing the future of manufacturing and supply chain," tech. rep., Chicago, 2017.

[39] Microsoft, "The Process Digital Twin: A step towards operational excellence," tech. rep.

[40] Deloitt, "Industry 4.0 and the digital twin: Manufacturing meets its match," tech. rep., Deloitte University Press, 2017.

[41] N. Mohammadi and J. E. Taylor, "Smart city digital twins," in Computational Intelligence (SSCI), 2017 IEEE Symposium Series on, pp. 1-5, IEEE, 2017.

[42] W. B. Rouse and N. Serban, "Understanding change in complex socio-technical systems," Information Knowledge Systems Management, vol. 10, no. 1-4, pp. 25-49, 2011.

[43] L. Anthopoulos, "Smart utopia VS smart reality: Learning by experience from 10 smart city cases," Cities, vol. 63, pp. 128-148, 2017.

[44] Nashvile MTA, "nMotion Nashville MTA Strategic Plan," 2018.

[45] T. Basar and G. J. Olsder, Dynamic noncooperative game theory, vol. 23. Siam, 1999.

[46] J. R. Marden, G. Arslan, and J. S. Shamma, "Cooperative control and potential games," IEEE Transactions on Systems, Man, and Cybernetics, Part B (Cybernetics), vol. 39, no. 6, pp. 1393-1407, 2009.

[47] D. Bollier, How smart growth can stop sprawl: a fledgling citizen movement expands. Washington, DC: Essential Books, 1998.

[48] N. Komninos, "The architecture of intelligent cities," in Conference Proceedings Intelligent Environments, vol. 6, pp. 5-6, IET, 2006.

[49] A. Cocchia, "Smart and digital city: A systematic literature review," in Smart city, pp. 13-43, Springer, 2014.

[50] Y.-T. C. Yang, "Building virtual cities, inspiring intelligent citizens: Digital games for developing students' problem solving and learning motivation," Computers \& Education, vol. 59, no. 2, pp. 365-377, 2012.

[51] S. Marvin, A. Luque-Ayala, and C. McFarlane, Smart urbanism: Utopian vision or false dawn? Routledge, 2015. 
[52] T. Nam and T. A. Pardo, "Smart city as urban innovation: Focusing on management, policy, and context," in Proceedings of the 5th international conference on theory and practice of electronic governance, pp. 185-194, ACM, 2011.

[53] S. Musterd and A. Murie, Making competitive cities. John Wiley \& Sons, 2011.

[54] S. Goldsmith and S. Crawford, The responsive city: Engaging communities through data-smart governance. John Wiley \& Sons, 2014.

[55] S. T. A. Pickett, M. L. Cadenasso, and J. M. Grove, "Resilient cities: meaning, models, and metaphor for integrating the ecological, socio-economic, and planning realms," Landscape and urban planning, vol. 69, no. 4 , pp. 369-384, 2004.

[56] L. Btgan, "Smart cities and sustainability models," Informatica Economic, vol. 15, no. 3, pp. 80-87, 2011.

[57] O. Söderström, T. Paasche, and F. Klauser, "Smart cities as corporate storytelling," City, vol. 18, no. 3, pp. 307-320, 2014.

[58] J. M. Shapiro, "Smart cities: quality of life, productivity, and the growth effects of human capital," The review of economics and statistics, vol. 88, no. 2, pp. 324-335, 2006.

[59] R. J. Sampson, "Urban sustainability in an age of enduring inequalities: Advancing theory and ecometrics for the 21st-century city," Proceedings of the National Academy of Sciences, vol. 114, no. 34, pp. 8957-8962, 2017.

[60] E. National Academies of Sciences and Medicine, Pathways to urban sustainability: Challenges and opportunities for the United States. National Academies Press, 2016. 